\title{
A Generalized Linear Modeling Approach for Characterizing Disease Incidence in a Spatial Hierarchy
}

\author{
W. W. Turechek and L.V. Madden
}

First author: Department of Plant Pathology, Cornell University, New York State Agricultural Experiment Station, Geneva 14456; and second author: Department of Plant Pathology, The Ohio State University, Ohio Agricultural Research Development Center, Wooster 44691.

Accepted for publication 28 October 2002.

\section{ABSTRACT}

Turechek, W. W., and Madden, L. V. 2003. A generalized linear modeling approach for characterizing disease incidence in a spatial hierarchy. Phytopathology 93:458-466.

Several statistical models are introduced to quantify the effect of heterogeneity on disease incidence relationships in a three-scale spatial hierarchy: the sampling unit level (highest), the leaf scale (intermediate), and the leaflet scale (lowest). The models are an extension of the theory previously developed for a two-scale hierarchy and were tested using data collected from strawberry leaf blight epidemics. Disease incidence at the sampling-unit scale (proportion of sampling units with one or more diseased leaflets) increased as a saturation-type curve with increasing leaflet or leaf disease incidence (proportion of leaflets or leaves diseased) as predicted by the good fit of the beta-binomial distribution to the leaflet and leaf data. The relationship could be accurately described, without curve fitting, by several simple nonlinear models, in which the aggregation of disease was represented by a modified binomial function incorporating an effective sample size that was either constant or dependent on mean incidence. The relationship between incidence at the leaflet and leaf scales could be modeled based on the combined sampling-unit models for leaflets and leaves. By taking the complementary $\log -\log$ (CLL) transformation of incidence, the equations could be expressed as generalized linear models, and curve fitting used to estimate the parameters. Generally, curve fitting gave slight to no improvement in the accuracy of the predictions of incidence. These models have broad applicability in sampling for disease incidence, and results can be used to interpret how diseased individuals at the lowest level in a hierarchy are arranged within sampling units.

Additional keyword: hierarchical sampling.
Quantifying spatial pattern or heterogeneity of disease incidence data generally requires that data be collected in the form of a cluster sample, in which sampling units are chosen in a random or systematic fashion (8), and all individuals within the sampling unit (e.g., leaflets) are categorized as diseased or healthy. A consequence of cluster sampling is that data are arranged hierarchically, thus providing disease information over a range of scales (9). The sampling unit (i.e., a collection of individuals [=subunits]) occupies the upper-most scale in the hierarchy, whereas the subunit occupies the lowest scale. In the simplest cluster sampling case, disease incidence can be assessed at two spatial scales, that is, at the scale of the subunits (e.g., leaves) and at the scale of the sampling units (e.g., plants).

Hughes et al. (9) characterized a theoretical relationship relating disease incidence between two hierarchical spatial scales for data collected in a cluster sample. Motivated by the theory of binomial sampling $(10,20,27)$, density-incidence-aggregation models in ecology $(18,19,21)$, and severity-incidence relationships (23), they showed that the proportion of sampling units (upper scale) diseased is a function of mean disease incidence at the scale of the subunits (lower scale) and the heterogeneity (dispersion) of disease incidence at the lower scale. The basis for this work centers on the statistical distribution of diseased incidence at the lower scale. With parameters of the distribution known, the zero term of the binomial or beta-binomial distribution is used to give the proportion of sampling units with no diseased individuals (23). Knowledge of the incidence-incidence relationship can be used to

Corresponding author: W. W. Turechek; E-mail address: wwt3@ cornell.edu

Publication no. P-2003-0218-01R

(C) 2003 The American Phytopathological Society help understand how diseased individuals are arranged relative to each other and their physical surroundings at various scales, what is sometimes called patch dynamics (12), and can be utilized in sampling. In the latter, one exploits the relation by observing only the disease status of the sampling units (are there one or more diseased individuals or not?) and predicts the variable of interest, that is, the proportion of diseased individuals (subunits). This forms the foundation of what Hughes and Gottwald (4) refer to as hierarchical sampling, which is closely related to the theory of group testing $(2,3,24)$.

Although there are elements of classic binomial sampling, density-incidence-aggregation relationships, and group testing in hierarchical sampling (9), the latter approach is unique. For instance, both binomial sampling and density-incidence-aggregation relationships are based on density of individuals (e.g., number of lesions per leaf or per plant) at the lower scale, whereas hierarchical sampling is based on incidence of disease (e.g., proportion of diseased leaves) at both scales. Moreover, group sampling is based on a binomial distribution of individuals at both scales, whereas hierarchical sampling is based on more relaxed assumptions of the distribution at the lower scale to account for disease aggregation.

It is straightforward to rearrange the binomial-based equation to predict the proportion of diseased individuals from the proportion of diseased sampling units (9). However, because of disease aggregation, most data sets are described much better by the betabinomial than the binomial distribution (25). Rearrangement of the beta-binomial to predict the proportion of diseased individuals generally is not possible, making direct use of hierarchical sampling more difficult. As an alternative approach, Madden and Hughes (15) showed that one can closely approximate the expression for the zero term of the beta-binomial with a binomial-type 
expression, which can then be manipulated to predict incidence at the lower scale. Another approach was adopted by Hughes and Gottwald (4,5), based on the work of Farrington (3), in which a generalized linear model is fitted to the two-scale incidence data. This approach does not depend explicitly on knowledge of the distribution of diseased individuals. In more complex situations, there may be multiple hierarchical scales of interest. For example, leaves within branches, branches within trees, and trees within fields. Recently shown by Turechek and Madden (26) for a threescale problem, there is no unique relationship in incidence between the lower and intermediate scales. To demonstrate, consider the study by Turechek and Madden (25) in which the incidence of Phomopsis leaf blight, caused by the fungal pathogen Phomopsis obscurans (13), was assessed in a three-scaled spatial hierarchy, leaflets, leaves, and sampling units. Sampling units consisted of 15 leaflets, collected as five leaves of three leaflets each. Although the proportion and heterogeneity of diseased leaflets (or leaves) uniquely determined the proportion of sampling units with disease (i.e., one or more diseased leaflets in a sampling unit), Turechek and Madden (26) showed that there could be many possible proportions of diseased leaves at a given proportion and heterogeneity of diseased leaflets. When the incidence of diseased leaflets was 0.4 , for example, and the heterogeneity parameter of the beta-binomial was described by the binary power law $(6,25)$, with parameters $b=1.14$ and $\ln \left(A_{x}\right)=0.88$, it is possible for incidence of diseased leaves to range from 0.46 to 0.85 for a field with 75 sampling units (Fig. 3A in Turechek and Madden [26]). Results for the intermediate scale depended on the withinsampling-unit arrangement of diseased leaflets among the leaves, conditional on the distributional properties of leaflet disease incidence. In general, the observed proportions of diseased leaves were low relative to their possible values, and were consistent with aggregating the diseased leaflets on nearly the smallest possible number of leaves within each sampling unit. For the example data set mentioned above, observed leaf incidence was 0.56 .

Because of the wide range of possible results for intermediate scales in a hierarchy, it is natural to ask whether it is possible to predict disease incidence between scales in a multiscale hierarchy. To answer this question, we extend the work of Hughes et al. (9), Hughes and Gottwald (4,5), and Madden and Hughes (15) by developing relatively simple models to quantify the relationship between incidence within a three-scaled spatial hierarchy and show what information is needed to quantify the relationship between scales. We work within the framework of generalized linear models (GLMs) to show how incidence relationships can be formulated for intermediate scales. The models developed provide the methodology needed to design hierarchical sampling plans (4) and can indicate the clustering process of plant diseases at various spatial scales.

\section{MATERIALS AND METHODS}

Data collection. Naturally occurring epidemics of Phomopsis leaf blight of strawberry (Fragaria $\times$ ananassa) were monitored over 2 years in several fields at two different commercial strawberry farms in Ohio (25). Details have been previously published. In brief, for each field and assessment date, a single row was randomly chosen and sampling units were selected approximately every $2.5 \mathrm{~m}$ along the row. Sampling units consisted of five leaves of three leaflets each, which were chosen without regard to disease status. Each leaflet was rated for the presence or absence of leaf blight symptoms. This was a form of cluster sampling in which the "clusters" (i.e., sampling units) were chosen systematically and all individuals (e.g., leaflets) per sampling unit $(n)$ were assessed (8).

Disease incidence could therefore be characterized at three scales in a spatial hierarchy: the leaflet (lowest) scale, $n=n_{t}=15$ (i.e., 15 leaflets per sampling unit); the leaf (intermediate) scale, $n=n_{f}=5$ (i.e., five leaves per sampling unit); and the samplingunit (highest) scale, $n=n_{s u}=1$. Note that we use a $t$ subscript for the lowest scale in the hierarchy (leafle $t$ ), $f$ subscript for the intermediate or middle scale in the hierarchy (leaf), and $s u$ subscript for the highest scale (sampling unit). If there was one or more Phomopsis lesion on a leaflet, then the leaflet was rated as diseased; if any leaflet in a leaf was diseased, then the leaf was rated as diseased; and if any leaflet (or leaf) in a sampling unit was diseased, the sampling unit was rated as diseased. At the sampling-unit scale, the cluster sampling reduces to a simple systematic sample of $N$ observations. The number of sampling units, $N$, varied from 49 to 106 , but was typically 75 . Several disease assessments were made in each field throughout the season. There were 67 data sets collected in 1996 and 54 in 1997.

Heterogeneity summary. In an earlier study, we characterized the spatial pattern of leaf blight using the 121 data described above (25). We showed that the beta-binomial distribution (14) fitted the disease incidence data better than the binomial distribution in $92 \%$ of the data sets when disease incidence was rated at the scale of the leaflets and in only $26 \%$ of the data sets when incidence was rated at the scale of the leaves (25). Parameters of the beta-binomial distribution are $p$ (specifically $p_{t}$ and $p_{f}$ for leaflets and leaves, respectively), the expected probability of an individual (leaflet or leaf) being diseased; and $\theta$ (specifically $\theta_{t}$ and $\theta_{f}$ ), the heterogeneity parameter, which equals 0 for a random pattern and increases without bound with increasing aggregation at the scale of the sampling unit or smaller. Mean leaflet disease incidence per data set, $\hat{p}_{t}$, ranged from 0.0009 to 0.78 , with a median across the 121 data sets of approximately 0.05 ; estimated heterogeneity parameter $\left(\hat{\theta}_{t}\right)$ ranged from 0.00 to 0.46 , with a median of 0.1 . Mean leaf disease incidence, $\hat{p}_{f}$, ranged from 0.0027 to 0.89 , with a median of approximately $0.01 ; \hat{\theta}_{f}$ ranged from 0.00 to 0.46 , with a median of 0.1 . Mean sampling-unit disease incidence, $\hat{p}_{s u}$, ranged from 0.013 to 1.0 , with a median of 0.35 . The binary form of Taylor's power law (6) was used as an assessment of heterogeneity across all data sets at both the leaflet and leaf scales.

Sampling-units diseased. The zero term $[\operatorname{Pr}(0)]$ and expected value (i.e., mean) of the probability distribution of diseased individuals can be used to characterize the theoretical relationship between the proportion of diseased sampling units and diseased individuals (9). If we let $\operatorname{Pr}_{t}(0)$ and $\operatorname{Pr}_{f}(0)$ be the probabilities that all leaflets and leaves in the sampling unit are disease free, respectively, then the probability that at least one leaflet is diseased per sampling unit is written as $p_{s u}=1-\operatorname{Pr}_{t}(0)$, and the probability that at least one leaf is diseased is $p_{s u}=1-\operatorname{Pr}_{f}(0)$. We first consider the relationship between $p_{s u}$ and $p_{t}$ and then consider leaves below. If the pattern of diseased leaflets is random, then the binomial distribution is reasonable for representing the frequency distribution of diseased leaflets per sampling unit. $\operatorname{Pr}_{t}(0)$ of the binomial distribution is given by $\left(1-p_{t}\right)^{n_{t}}$. When $n_{t}$ is fixed, then the moment estimate of $p_{t}$ is given by

$$
\hat{p}_{t}=\left(\sum_{i} \hat{p}_{t, i}\right) / N
$$

in which $i=1,2, \ldots N$, and $\hat{p}_{t, i}$ is the proportion of diseased leaflets in the $i$ th sampling unit. If diseased leaflets have an aggregated pattern, the beta-binomial distribution may be appropriate for representing the frequency distribution of diseased leaflets (9). $\operatorname{Pr}_{t}(0)$ is then given by

$$
\operatorname{Pr}_{t}(0)=\prod_{j=0}^{n_{t}-1} \frac{1-p_{t}+j \theta_{t}}{1+j \theta_{t}}
$$

in which $\Pi(\bullet)$ represents the product function and $j$ is a counting index. When $\theta_{t}=0$, equation 2 reduces to the zero term of the binomial distribution. 
When incidence at the lower scale is described by the binomial distribution, $p_{s u}$ is estimated as

$$
\tilde{p}_{s u}=1-\left(1-\hat{p}_{t}\right)^{n_{t}}
$$

A tilde $(\sim)$ is used to denote an estimate that has been derived from measurements made at scale other than the one indicated by its subscript. A direct estimate of $p_{s u}\left(\hat{p}_{s u}\right)$ at the sampling-unit scale would be the proportion of sampling units with one or more diseased leaflets. When the frequency distribution of diseased leaflets follows a beta-binomial distribution, the estimate of $p_{s u}$ is

$$
\widetilde{p}_{s u}=1-\prod_{j=0}^{n_{t}-1} \frac{1-\hat{p}_{t}+j \hat{\theta}_{t}}{1+j \hat{\theta}_{t}}
$$

If $\hat{\theta}_{t}$ varies with $\hat{p}_{t}$ and the binary power law (9) describes the variance data, as previously demonstrated for strawberry leaf blight $(25), \hat{\theta}_{t}$ can be predicted using

$$
\hat{\theta}_{t}=\frac{\hat{a}_{t}-f\left(\hat{p}_{t}\right) / n_{t}}{f\left(\hat{p}_{t}\right)-\hat{a}_{t}}
$$

in which $\hat{a}_{t}$ and $f\left(\hat{p}_{t}\right)$ are determined from the binary power law. Specifically, $\hat{a}_{t}=\hat{A}_{x, t} \cdot n_{t}^{\hat{b}_{t}-2}, f\left(\hat{p}_{t}\right)=\left(\hat{p}_{t} \cdot\left[1-\hat{p}_{t}\right]\right)^{1-\hat{b}_{t}}$, and $\hat{A}_{x, t}$ and $\hat{b}_{t}$ are parameters estimated from the binary power law when counts of diseased leaflets (e.g., 0 to 15) have been analyzed (25). The binary power law can be written as

$$
\ln \left(v_{\mathrm{obs}, t}\right)=\ln \left(\hat{A}_{x, t}\right)+b_{t} \cdot \ln \left[n_{t} \hat{p}_{t}\left(1-\hat{p}_{t}\right)\right]
$$

where $v_{\mathrm{obs}, t}$ is the observed (calculated) variance of $x_{t}$, and $n_{t} \hat{p}_{t}\left(1-\hat{p}_{t}\right)$ is the expected binomial variance of diseased leaflets $\left(v_{\text {bin }, t}\right)$.

Equation 4 (or 2) is not easily manipulated or rearranged to allow prediction of $p_{t}$ from $p_{s u}(15)$. Such a prediction would be useful when one has information (possibly from a biochemical diagnostic test) on the pooled $n_{t}$ individuals of each sampling unit, and then wishes to use this information to estimate $p_{t}$. Alternative methods to determine $\tilde{p}_{s u}$ rely on modifications of equation 3 and empirical descriptions of heterogeneity. One approach is to follow Rao and Scott (22) and use the so-called "design effect" or deff, as termed in the survey sampling literature (11), to correct for spatial heterogeneity (or overdispersion) of binary data. The deff is simply the ratio of $v_{\mathrm{obs}, t}$ to $v_{\mathrm{bin}, t}$, and represents the inflation of the observed variance due to overdispersion (i.e., spatial heterogeneity at some scale). In the context of spatial pattern analysis, the deff is equivalent to the index of dispersion, $D$, and is calculated at the leaflet scale by

$$
D_{t}=\frac{v_{\mathrm{obs}, t}}{v_{\mathrm{bin}, t}}=\frac{\sum_{i}\left(x_{t, i}-n_{t} \hat{p}_{t}\right)^{2} /(N-1)}{n_{t} \hat{p}_{t}\left(1-\hat{p}_{t}\right)}
$$

$D_{t}$ can also be written as $\left(1+n_{t} \theta_{t}\right) /\left(1+\theta_{t}\right)$ if the beta-binomial distribution adequately characterizes the spatial pattern of $p_{t}$. Then, one can define

$$
n_{D, t}=n_{t} / D_{t}
$$

which is known as the "effective sample size" in the sampling literature (11). The effective sample size represents the reduction in information obtained in the sample due to the effects of overdispersion (spatial pattern at some scale). When there are several data sets, an average value of their $D_{t}$ 's could be used in equation 8. In this context, we assume $D_{t}$ is not systematically related to $p_{t}$. However, if $b_{t}>1$ in the binary power law (equation 6), then there is a systematic relationship between $D_{t}$ and $p_{t}(7)$, and an effective sample size $\left(n_{\delta, t}\right)$ can be written as

$$
n_{\delta, t}=f\left(\hat{p}_{t}\right) / \hat{a}_{t}
$$

in which $f\left(\hat{p}_{t}\right)$ and $\hat{a}_{t}$ are defined above. Equation 9 is derived by predicting $D_{t}$ from $p_{t}$ using the binary power law and substituting this expression for $D_{t}$ in equation 8 .

Recently, Madden and Hughes (15) developed an iterative and graphic method to determine another type of effective sample size $\left(n_{v, t}\right)$ when data are known to be beta-binomially distributed; the method is based on numerically approximating equation 4 with a binomial-type expression. This third effective sample size can also be predicted with the approximation

$$
\begin{gathered}
n_{\mathrm{v}, t}=n_{t} /\left(0.98135+0.8179 \theta_{t}+0.004958 n_{t}+0.30387 \theta_{t} n_{t}-\right. \\
\left.0.3471 \theta_{t}^{2}-0.08475 \theta_{t}^{2} n_{t}\right)
\end{gathered}
$$

In equation $10, \theta_{t}$ can be obtained either by averaging values over all the data sets (assuming, in effect, constant heterogeneity) or by using equation 5 when $b_{t}$ of the binary power law is greater than 1 (i.e., variable heterogeneity) (7). We label a general effective sample size as $n_{\text {. }}$, and when specifically applied to the leaflet or leaf scale as $n_{\bullet, t}$ and $n_{\bullet, f}$, respectively.

To correct for overdispersion, $n_{\bullet, t}\left(n_{D, t}, n_{\delta, t}\right.$, or $\left.n_{v, t}\right)$ replaces $n_{t}$ in equation 3 , giving the general expression

$$
\tilde{p}_{s u}=1-\left(1-\hat{p}_{t}\right)^{n_{*}, t}
$$

Note that no curve fitting of the $\hat{p}_{s u}: \hat{p}_{t}$ relationship is necessary to use equation 11 once $\hat{p}_{t}$ and heterogeneity of disease, as quantified by $n_{D, t}, n_{\delta, t}$, or $n_{v, t}$, are known (or assumed).

Farrington (3) showed that the binomial equation 3 can be formulated into the framework of a generalized linear model (1), thus, allowing for direct curve fitting (parameter estimation) of incidence between two scales and explicit consideration of the binomial error structure of $\hat{p}_{s u}$ (but not necessarily $\hat{p}_{t}$ ). Let CLL denote the complementary log-log function of a random variable $z, \operatorname{CLL}(z)=\ln [-\ln (1-z)]$. Algebraic rearrangement of equation 11 for the effective sample size model leads to

$$
\operatorname{CLL}\left(\widetilde{p}_{s u}\right)=\ln \left(n_{\bullet, t}\right)+\operatorname{CLL}\left(\hat{p}_{t}\right)
$$

This is an equation for a straight line with slope 1 and intercept of $\ln \left(n_{\bullet, t}\right)$. Although equation 12 can be used with no curve fitting (i.e., no parameter estimation for the $p_{s u}: p_{t}$ relationship) as a reformulation of equation 11 , it can also be used as a statistical model for $\operatorname{CLL}\left(\widetilde{p}_{s u}\right)$ in relation to $\operatorname{CLL}\left(\hat{p}_{t}\right)$ with unknown intercept, $\ln \left(n_{\bullet, t}\right)$, and known slope of 1 . In this case, $n_{\bullet, t}$ is not necessarily the effective sample size as defined by Rao and Scott (22) but a parameter describing the $p_{s u}: p_{t}$ relationship. In GLIM (Generalized Linear Interactive Modeling, release 3.77, Numerical Algorithms Group, England), $\ln \left(n_{\bullet, t}\right)$ can be estimated with a fixed slope by treating $\operatorname{CLL}\left(\hat{p}_{t}\right)$ as an "offset" variable (1).

Equation 12 with $n_{\bullet, t}$ given by $n_{D, t}$ (equation 8 ) or $n_{v, t}$ (equation 10) assumes constant degree of overdispersion for all data sets when a single (average) $D_{t}$ or $\theta_{t}$, respectively, is used to obtain $n_{D, t}$ or $n_{v, t}$. Equation 12 can also be used under the assumption of variable heterogeneity by predicting $\theta_{t}$ with equation 5 and using these predictions to obtain $n_{v, t}$ (equation 10) at each $\hat{p}_{t}$.

As an alternative, if overdispersion varies with $\hat{p}_{t}$ according to the binary power law (equation 6), then equation 9 for $n_{\delta, t}$ can be directly substituted for $n_{\bullet, t}$ in equation 12 , giving (after some algebraic manipulation)

$$
\operatorname{CLL}\left(\tilde{p}_{s u}\right)=-\ln \left(a_{t}\right)+\left(1-b_{t}\right) \cdot \ln \left[\hat{p}_{t}\left(1-\hat{p}_{t}\right)\right]+\operatorname{CLL}\left(\hat{p}_{t}\right)
$$

Equation 13 can be utilized with no curve fitting by using $\hat{a}_{t}$ and $\hat{b}_{t}$ from the power law results at the leaflet scale or with curve 
fitting (parameter estimation). With curve fitting, $\operatorname{CLL}\left(\hat{p}_{t}\right)$ is treated as an "offset", $\ln \left[\hat{p}_{t}\left(1-\hat{p}_{t}\right)\right]$ is treated as the independent variable (predictor) with slope parameter $1-b_{t}$, and intercept parameter $-\ln \left(a_{t}\right)$. When $a_{t}$ and $b_{t}$ are estimated through direct curve fitting of the $\hat{p}_{s u}: \hat{p}_{t}$ relationship, their meaning is analogous to, but not the same as, $\hat{a}_{t}$ and $\hat{b}_{t}$ in equations 6 and 9. Equation 12 can be generalized in another way by assuming a slope other than 1 (4). That is, $\operatorname{CLL}\left(\hat{p}_{t}\right)$ is replaced by $\beta \operatorname{CLL}\left(\hat{p}_{t}\right)$, in which $\beta$ is an unknown parameter. This approach was not taken here because one then cannot interpret the model in terms of an effective sample size.

Equation 3 (binomial) and equation 4 (beta-binomial) can also be written with CLLs on the left hand side. For the binomial,

$$
\operatorname{CLL}\left(\tilde{p}_{s u}\right)=\ln \left(n_{t}\right)+\operatorname{CLL}\left(\hat{p}_{t}\right)
$$

and for the beta-binomial,

$$
\operatorname{CLL}\left(\tilde{p}_{s u}\right)=\ln \left[-\ln \prod_{j=0}^{n_{t}-1}\left(\frac{1-\hat{p}_{t}+j \theta_{t}}{1+j \theta_{t}}\right)\right]
$$

However, with equation 15, the predictor variable is a complicated product function and the intercept is zero; that is, the predictor is not a CLL function. The CLL-function approach was recently used by Xu and Madden (28) for representing incidence-incidence and incidence-density relationships for apple powdery mildew.

Prediction comparisons. A generalized linear modeling approach was used to evaluate the accuracy of predictions of $p_{s u}$ (i.e., $\tilde{p}_{s u}$ ) from $\hat{p}_{t}$. Five cases without curve fitting of the $p_{s u}: p_{t}$ relationship, and two cases of curve fitting, are summarized.

The cases with no curve fitting are (i) random (binomial) distribution of diseased leaflets (equation 14); (ii) beta-binomial distribution for diseased leaflets (equation 15); (iii) effective sample size of Kish (11) and Rao and Scott (22) calculated using mean $D_{t}$ (equation 12, using $n_{D, t}$ [equation 8] for $n_{\bullet, t}$ ); (iv) effective sample size of Kish (11) calculated for variable heterogeneity described by binary power-law parameters (equation 12 , using $n_{\delta, t}$ [equation 9] for $n_{\bullet, t}$ ); and (v) effective sample size of Madden and Hughes (15) calculated for variable heterogeneity (equation 12, using $n_{v, t}$ [equation 10] for $n_{\bullet, t}$, with $\theta_{t}$ quantified by equation 5).

Curve fitting (parameter estimation) was done for the following cases: (vi) equation 12 (i.e., estimated $\ln \left(n_{\bullet, t}\right)$; compare this with iii); and (vii) equation 13 (i.e., estimated $-\ln \left(a_{t}\right)$ and $1-b_{t}$; compare with iv and $\mathrm{v}$ ). The binomial distribution was assumed for $\hat{p}_{s u}$ in curve fitting, because each sampling unit was classified as being diseased or not (9).

For all seven cases, the deviance was calculated for assessing goodness of fit. The deviance is analogous to the residual (error) sum of squares with normal-theory regression. For situations i to $\mathrm{v}$, in which there were no parameters to estimate, the GLIM method of Aitkin et al. (page 177 of literature citation 1) was used to determine deviances.

The seven cases (models) described previously were evaluated for each year separately. Then, the analyses were repeated for all seven cases to model the relationship between $\hat{p}_{s u}$ and disease incidence at the leaf (intermediate) scale $\left(\hat{p}_{f}\right)$. That is, $\hat{p}_{f}$ was treated as the lower scale (ignoring leaflet information). In the case of leaves: $\hat{p}_{f}, \hat{\theta}_{f}, n_{f}, D_{f}, \hat{a}_{f}, \hat{b}_{f}, n_{D, f}, n_{\delta, f}$, and $n_{v, f}$ were substituted for the corresponding leaflet terms ( $t$ subscript) into equations 1 to 15 . Thus, four data sets in total were evaluated in this manner: leaflets, 1996; leaflets, 1997; leaves, 1996; and leaves, 1997. Use of these symbols without an $f$ or a $t$ subscript indicates that we are referring to the general term that could apply to leaflets or leaves.

Leaflets-leaves diseased. The observed relationship between incidence of diseased sampling units $\left(p_{s u}\right)$ and diseased leaflets $\left(p_{t}\right)$, and between $p_{s u}$ and diseased leaves $\left(p_{f}\right)$, can be used as the foundation to model disease incidence between $p_{t}$ and $p_{f}$. The methods here are worked through for the situation when information is known on all three scales. This allows the development of an approach that, depending on the results, could be used when the leaf and/or sampling-unit incidence was not known (e.g., when there is a pooling of individuals [subunits] and a biochemical assay performed on the pooled data, ultimately predicting the incidence at the lowest scale). Starting with the binomial-based equation 3, and considering the theoretical probabilities rather than the estimates, $p_{s u}$ is given both by $1-\left(1-p_{t}\right)^{n_{t}}$ and $1-\left(1-p_{f}\right)^{n_{f}}$. Equating these two expressions leads to

$$
p_{f}=1-\left(1-p_{t}\right)^{n_{t} / n_{f}}
$$

If one used the estimates of $p_{t}\left(\hat{p}_{t}\right)$ in equation 16 , we write the estimate of $p_{f}$ as $\left(\widetilde{p}_{f}\right)$, resulting in

$$
\tilde{p}_{f}=1-\left(1-\hat{p}_{t}\right)^{n_{t} / n_{f}}
$$

As before, we can correct for overdispersion at the leaflet scale by substituting $n_{\bullet, t}$ and $n_{\bullet, f}$ for $n_{t}$ and $n_{f}$, respectively,

$$
\widetilde{p}_{f}=1-\left(1-\hat{p}_{t}\right)^{n_{\bullet, t} / n_{\bullet}, f}
$$

in which these effective sample sizes can be constant or a function of $p_{t}$ and $p_{f}$. CLL versions of these can be written for equations 17 and 18 , with $n_{t} / n_{f}$ or $n_{\bullet, t} / n_{\bullet, f}$ in place of $n_{t}$ or $n_{\bullet, t}$ in equations 14 and 12 , respectively.

$$
\operatorname{CLL}\left(\widetilde{p}_{f}\right)=\ln \left(n_{\bullet, t} / n_{\bullet}, f\right)+\operatorname{CLL}\left(\hat{p}_{t}\right)
$$

The new exponents could be obtained based on analysis strictly at the respective scale (e.g., binary power-law results for $\hat{p}_{t}$ ) or based on curve fitting of $\operatorname{CLL}\left(\widetilde{p}_{f}\right)$ in relation to $\operatorname{CLL}\left(\hat{p}_{t}\right)$.

Four of the several possible variations of equations 17 and 18 are demonstrated for determining $\widetilde{p}_{f}$, labeled cases viii to xi. For case viii, we assumed that data were random (i.e., binomial; equation 3) at both the leaflet and leaf scales (equation 17). This gives a nominal value of $n_{t} / n_{f}=3$. For case ix, leaf disease incidence was assumed to be random (i.e., binomial; equation 3) and leaflet disease incidence was assumed to be described by the beta-binomial distribution with heterogeneity quantified by the effective sample size of Madden and Hughes (15), $n_{v, t}$. Thus, in equation $18, n_{v, t}$ was used for $n_{\bullet, t}$, and $n_{f}$ was used for $n_{\bullet, f}$. Here, $n_{v, t}$ was calculated with equation 10 , using equation 5 for $\theta_{t}$ with the respective $a$ and $b$ parameters determined from the previous single-scale analysis (25). For case $\mathrm{x}$, incidence was assumed to be described by the beta-binomial distribution, with heterogeneity varying at the leaflet according to $n_{v, t}$ (same as case ix) and constant heterogeneity at the leaf scale using the effective sample size defined in equation $8\left(n_{D, f}\right)$. Using the method of Aitkin et al. (1), the deviances for the three non-curve fitting cases were determined (note that no parameters are estimated using this approach). In case $\mathrm{xi}$, we used curve fitting to estimate the intercept parameter $\ln \left(n_{t} / n_{f}\right)$ in equation 19 and assumed a constant slope of 1 .

\section{RESULTS}

Sampling units. Figure 1 shows the relationship between the proportion of sampling units with Phomopsis blight $\left(\hat{p}_{s u}\right)$ and the proportion of diseased leaflets $\left(\hat{p}_{t}\right)$ (Fig. 1A and B) or diseased leaves $\left(\hat{p}_{f}\right)$ (Fig. 1C and D) for the data of 1996 and 1997. Curves are based on the binomial model (case i, equation 14), beta-binomial model (case ii, equation 15), effective sample size based on Madden and Hughes (15) (case v, equation 10), and one curve fitting method (case vii, equation 13 [variable heterogeneity]). Table 1 shows the deviances for all the methods of predicting $p_{s u}$ from $\hat{p}_{t}$ or $\hat{p}_{f}$. The binomial case provided the poorest 
fit to the data in both years for both leaflets and leaves, whereas the beta-binomial case provided very good predictions of the data (Table 1; Fig. 1). The latter was expected because of the good fit of the beta-binomial distribution to the data (25).

A significant improvement in prediction compared with the binomial case could be accomplished by replacing $n$ with Rao and Scott's (22) effective sample size, $n_{D}$ or $n_{\delta}$ (equations 8 and 9) or Madden and Hughes' (15) effective sample size, $n_{v}$ (equation 10) (Fig. 1; Table 1). (It is re-emphasized here that symbols used without the $t$ or $f$ subscript can refer to either hierarchical scale). The values of $n_{D}, n_{\delta}$, and $n_{v}$ used in the predictions were determined with the estimated parameters of the binary power law reported in Turechek and Madden (25) and shown in Figure 2. Values of $n_{v}$ were larger than the other effective sample sizes, as expected based on the results in Madden and Hughes (15). Even for the effective sample sizes that depended on disease incidence, there was little change in values between incidences of 0.2 and 0.8 . Moreover, the effective samples sizes for diseased leaves changed little with incidence because the estimated $b_{f}$ was close to $1.0(25)$.

Using $n_{D}$ (based on a mean $D$; case iii) resulted in a generally better fit than using $n_{\delta}$ (which was based on equation 9 for variable heterogeneity [ $\theta$ or $n$.]; case iv). Of the three effective sample size-based models, Madden and Hughes' (15) method (case v) clearly fitted the data best, especially at the leaflet scale.
In fact, in three of the four data sets, the use of $n_{v, t}$ produced closer predictions of $\hat{p}_{s u}$ than one of the curve fitting approaches (case vi). However, of all the non-curve-fitting cases, the beta-binomial model (case ii) best described the relationship between $\hat{p}_{s u}$ and $\hat{p}_{t}$ or $\hat{p}_{f}$, but this model cannot be inverted for easy manipulations (9).

There generally was a linear relationship between the CLL function of incidence at the sampling unit and lower scales; an example is shown in Figure 3A. Direct curve fitting to estimate parameters $n$. or $A_{x}$ and $b$ (to then obtain $n$. as a function of $\hat{p}$ ) generally did not appreciably improve the predictions of $\hat{p}_{s u}$ (Table 1; cases vi and vii) compared with beta-binomial predictions (i.e., case ii [equation 15 and no parameter estimation]). In fact, the beta-binomial equation (with no curve fitting) had a lower deviance for leaf incidence data in both years, although not significantly so. Only for leaflet data in 1997 was the deviance for curve fitting (case vii) significantly lower than the deviance for the beta-binomial model.

Leaves and leaflets. The relationship between the observed proportion of diseased leaflets and leaves is shown in Figure 4A and $\mathrm{B}$ with predictions based on models for diseased sampling units. Curves represent the theoretical relationship for the binomial distribution (case viii; equation 17), two adjustments for heterogeneity, one at just the leaflet scale (case ix) and the other at both scales (case $\mathrm{x}$ ), and one based on curve fitting the $\hat{p}_{t}: \hat{p}_{f}$

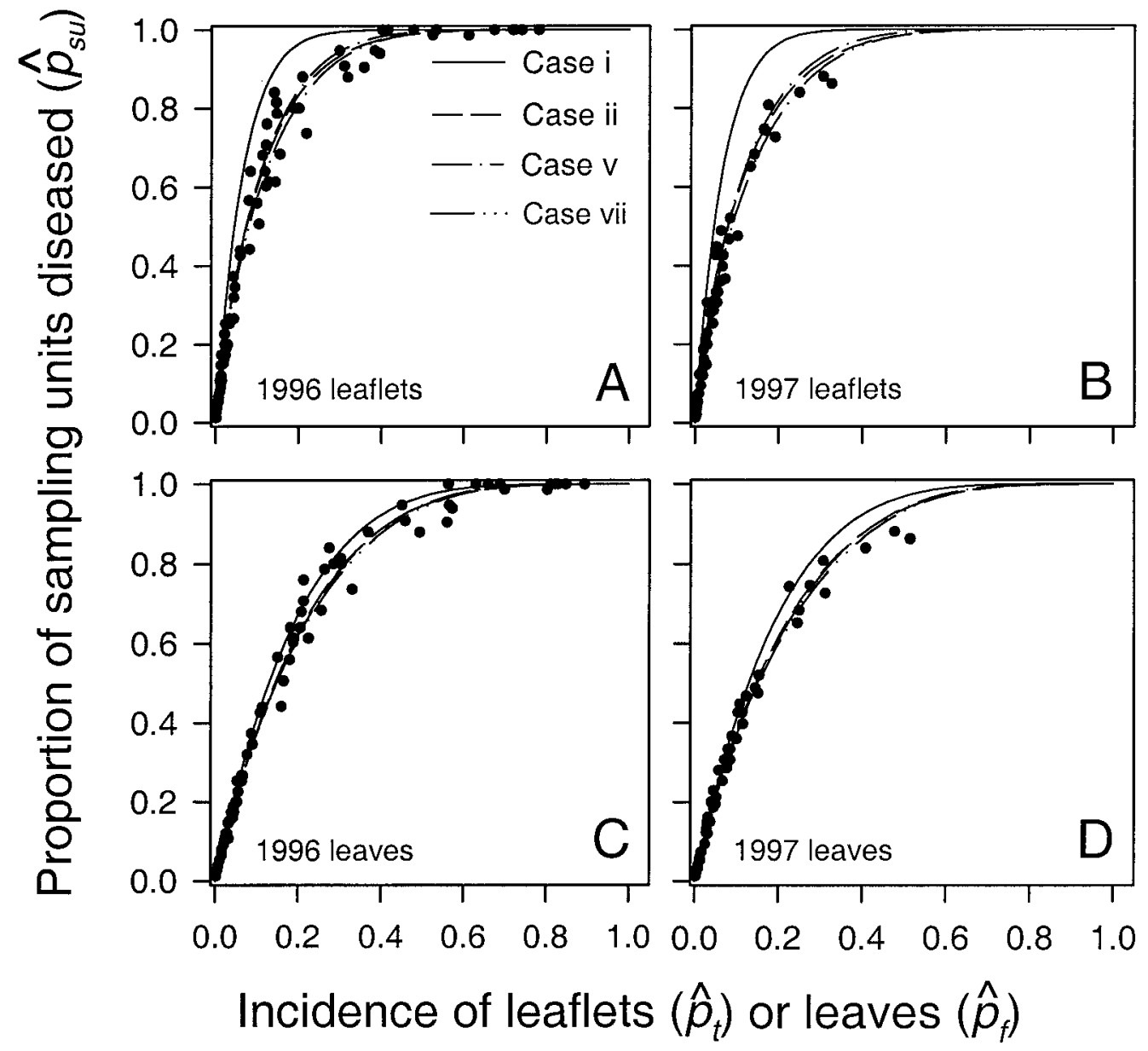

Fig. 1. Relationships between estimated parameters of the beta-binomial distribution at the lowest (leaflet; $t$ ) and intermediate (leaf; $f$ ) scales, representing probability of disease $\left(\hat{p}_{t}\right.$ or $\left.\hat{p}_{s u}\right)$, and the estimated binomial probability that a sampling unit has one or more diseased leaflets $\left(\hat{p}_{s u}\right)$. Individual graphs report A, $\hat{p}_{t}$ versus $\hat{p}_{s u}$ in 1996; B, $\hat{p}_{t}$ versus $\hat{p}_{s u}$ in 1997; C, $\hat{p}_{f}$ versus $\hat{p}_{s u}$ in 1996; and $\mathbf{D}, \hat{p}_{f}$ versus $\hat{p}_{s u}$ in 1997. Points represent single assessments of Phomopsis leaf blight in strawberry fields, and lines represent three prediction methods. Case i (solid line) is derived from the binomial distribution (equation 14); case ii (broken line) is derived from the beta-binomial distribution with $\hat{\theta}_{t}$ (or $\hat{\theta}_{f}$ ) defined as a function of disease incidence at the leaflet (or leaf) level (equation 15); case $\mathrm{v}$ (dash-dot line) is derived using Madden and Hughes' (15) effective sample size with $\hat{\theta}_{t}$ (or $\hat{\theta}_{f}$ ) being considered a function of incidence (equation 10, with $\hat{\theta}_{t}$ from equation 5); and case vii (dash-dot-dot line) is derived from the binomial function with $n_{t}$ replaced by a function of $\hat{p}_{t}$ (or $\hat{p}_{f}$ ), based on the binary power law (equation 13 ), with parameters $-\ln (a)$ and $1-b$ estimated using ordinary least squares curve fitting. 
relationship (case xi). With no correction for heterogeneity, there was systematic overprediction of $\hat{p}_{f}$ (Fig. 4; case viii). Predictions were substantially improved when corrections were made for heterogeneity at either one or both of the scales using Madden and Hughes' (15) effective sample size with variable heterogeneity ( $n_{v, t}$ [equation 10] with $\theta_{t}$ predicted with equation 5). In 1996, the best fitting model resulted when heterogeneity at the leaflet scale was described by $n_{v, t}$ and at the leaf scale heterogeneity was assumed to be binomial (i.e., $n_{f}$; case ix). Deviances for cases viii, ix, $x$, xi in 1996 were 2,669, 364, 513, and 410 (df $=67$ or 66 [case xi]), respectively. As expected based on the results for the relationships between incidence at the sampling unit scale and each of the two lower scales, there was a linear relationship between $\operatorname{CLL}\left(\hat{p}_{f}\right)$ and $\operatorname{CLL}\left(\hat{p}_{t}\right)$ (Fig. 3B); however, curve fitting of the $\hat{p}_{f}: \hat{p}_{t}$ relationship (case xi) did not result in a better fit than found for case ix.

In 1997, curve fitting provided the best fit (case xi). The best fitting model that did not involve direct curve fitting of the relationship resulted when heterogeneity at the leaflet scale was described by $n_{v, t}$ and at the leaf scale heterogeneity was assumed to be binomial (i.e., $n_{f}$; case ix). However, the results were very similar for cases ix and x. Deviances for the 1997 data were 675, 62,80 , and 46 for cases viii, ix, $\mathrm{x}$, and $\mathrm{xi}$, respectively ( $\mathrm{df}=54$ or 53 [case xi only]). The overall lower deviances for the 1997 predictions was likely a result of the smaller range of observed incidences in 1997 compared with 1996. Other pairs of prediction cases (i to vii) at the leaf and leaflet scale could be used for predicting $\hat{p}_{f}$ from $\hat{p}_{t}$, but the four situations chosen here summarize the range of results.

\section{DISCUSSION}

As expected from several studies $(4,5,9,15)$, the incidence of diseased sampling units $\left(\hat{p}_{s u}\right)$ increased with increasing incidence of diseased individuals per sampling unit, either leaflets $\left(\hat{p}_{t}\right)$ or leaves $\left(\hat{p}_{f}\right)$, as saturation-type curves. That is, $\hat{p}_{s u}$ increased rapidly at low $\hat{p}_{t}$ (or $\hat{p}_{f}$ ), and the rate of increase declined monotonically to zero as $\hat{p}_{s u}$ approached one. This relationship, which is a function of individuals per sampling unit, $n_{t}$ (or $n_{f}$ ), and heterogeneity of diseased individuals among sampling units, was well predicted by the beta-binomial-based model introduced by Hughes et al. (9). Because of the good fit of the data by the beta-binomial distribution, it was possible to predict with high accuracy sampling-unit disease incidence $\left(\hat{p}_{s u}\right)$ without any curve fitting, based only on disease incidence (either $\hat{p}_{t}$ or $\hat{p}_{f}$ ) and heterogeneity ( $\hat{\theta}_{t}$ or $\hat{\theta}_{f}$ ) at the lower scale (equations 4 and 15; Fig. 1).

The complicated beta-binomial-based model could also be approximated by an equation of the binomial form by replacing $n_{t}$ or $n_{f}$ with parameters $n_{\bullet, t}$ or $n_{\bullet, f}$, respectively (defined by equations 8 to 10 ; Fig. 2). Of the various methods for determining $n_{\bullet, t}$ or $n_{\bullet, f}$ without curve fitting of the $\hat{p}_{s u}: \hat{p}_{t}$ relationship, the approach of Madden and Hughes (15) was the most accurate (case v in Table 1; Fig. 1). Our results also confirm the theoretical work of Madden and Hughes (15) that the standard effective sample size of Rao and Scott (22) and Kish (11) (labeled $n_{D, t}$ or $n_{D, f}$ here), which has many beneficial statistical uses for overdispersed binary data, is not directly useful for predicting disease incidence in a spatial hierarchy (cases iii and iv in Table 1). The exception would be for small $n$ (e.g., $n \lesssim 4$ ), where $n_{v} \approx n_{D}$ (15). It should be noted that the effective sample size of Rao and Scott (22) is based on the premise that one can obtain the mean and variance for an overdispersed (i.e., beta-binomial) distribution with a binomial distribution model by modifying the constant for number of individuals. This modification, however, was not developed to allow one to duplicate the zero term of the beta-binomial with a binomial function. On the other hand, the effective sample size of Madden and Hughes (15) was developed specifically to obtain the zero term of the beta-binomial with a binomial function. Although the best predictions of $p_{s u}$ were obtained by one curve fitting method-that is, by treating $A_{x, t}$ and $b_{t}$ (or $A_{x, f}$ and $b_{f}$ ) in equation 13 as unknown parameters and estimating these parameters for the $\hat{p}_{s u}: \hat{p}_{t}$ (or $\hat{p}_{s u}: \hat{p}_{f}$ ) data using generalized linear models (case vii) - the improvement in accuracy over case $\mathrm{v}$ was only minor. In fact, when one considers the loss of 2 df by estimating these parameters, there is little evidence of an improvement of fit through curve fitting.

As shown by Madden and Hughes (15), assuming the betabinomial is the appropriate distribution for disease incidence at the lower scale, then the hierarchical scale models of cases iii to vii are all approximations because these models cannot exactly duplicate the hierarchical scale relationship of the beta-binomial distribution. In particular, to duplicate equation 2 with a binomialtype function for even fixed heterogeneity (i.e., same $\theta_{t}$ for all data sets), $1-p_{t}$ would have to be raised to a complicated summation function involving $p_{t}, \theta_{t}$, and $n_{t}$ (equation 11 in

TABLE 1. Deviances (Dev.) for the agreement between observed and predicted number of sampling units with diseased individuals $\left(\hat{p}_{s u}\right)$ based on either the proportion of leaflets $\left(\hat{p}_{t}\right)$ or leaves $\left(\hat{p}_{f}\right)$ with symptoms of Phomopsis leaf blight of strawberry

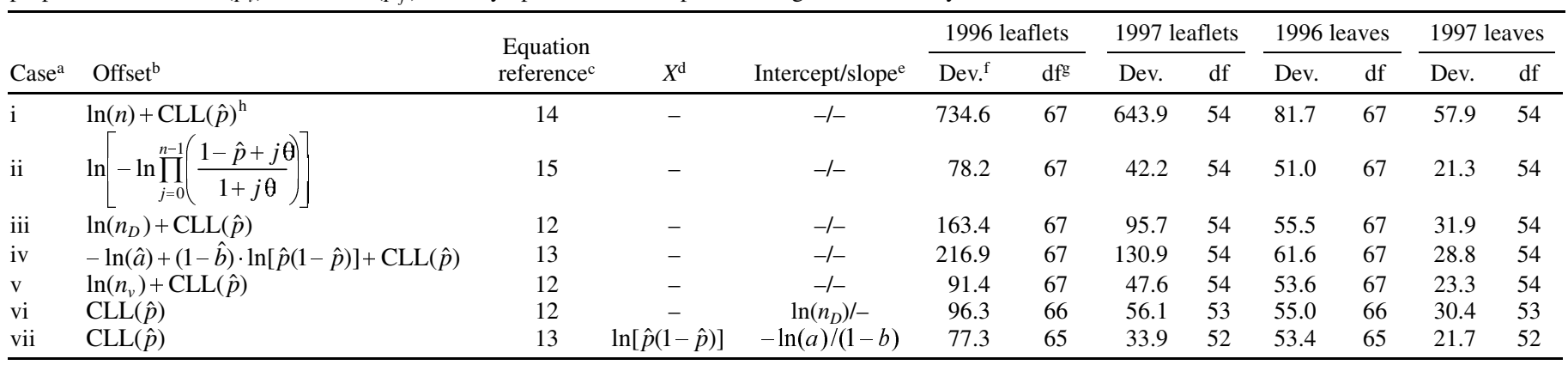

${ }^{a}$ Roman numeral refers to cases as discussed in text.

b GLIM terminology; these terms are considered to be known.

${ }^{\mathrm{c}}$ Equation reference as numbered in text.

d Independent variable (predictors); "-" = no independent variable used in model (thus, no parameters estimated).

e Values of the slope and intercept; " $-"=$ no slope or intercept estimated.

${ }^{f}$ Small values of Dev. generally reflect a good fit. For binomial data, values of Dev. less than or equal to degrees of freedom indicate a good fit. A model is considered to fit the data better than a competing model (if models are nested so that one of the models in the comparison is a special case of the other) if the reduction in deviance is greater than $\chi_{v}^{2}$, in which $\chi_{v}^{2}$ is chi-square deviate with $v$ degrees of freedom, where $v=\mathrm{df}_{1}-\mathrm{df}_{2}$ is the absolute difference of the degrees of freedom between competing models. No formal test is possible if $\mathrm{df}_{1}=\mathrm{df}_{2}$.

g Degrees of freedom.

${ }^{\text {h }} \operatorname{CLL}(\hat{p})=$ complimentary $\log -\log$ function $\{\ln [-\ln (1-p)]\}$. 
Madden and Hughes [15]). A CLL plot (Fig. 3) is one useful way of visualizing the approximation-models based on raising $1-p_{t}$ to a constant (such as equation 11 in this manuscript) result in an exact straight-line relationship in a CLL plot (equation 12), but a beta-binomial distribution (equation 17) results in slight curvature in the CLL plot at very high values of $p_{t}$ (Fig. 1 in Madden and Hughes [15]). However, when $n_{v, t}$ is properly chosen based on the heterogeneity of disease incidence, the approximation comes very close to the true beta-binomial values over most of the range of incidence. With the data sets in this study, the curvature in the CLL plot (Fig. 3) was not apparent because there were no values of $\hat{p}_{t}>0.9$.

We were able to use the modified binomial functions to predict $\hat{p}_{f}$ from $\hat{p}_{t}$ (equation 18). As with the $\hat{p}_{s u}: \hat{p}_{t}\left(\right.$ or $\hat{p}_{s u}: \hat{p}_{f}$ ) relationship, the effects of disease heterogeneity could be accounted for using $n_{v, t}$ or $n_{v, f}$, as was done in cases ix and x. Use of $n_{v, t}$ or $n_{v, f}$, either as single numbers or as functions of $\hat{p}_{t}$ or $\hat{p}_{f}$, to correct for the effects of heterogeneity between two scales is strictly empirical. Nonetheless, the use of the modified binomial-based
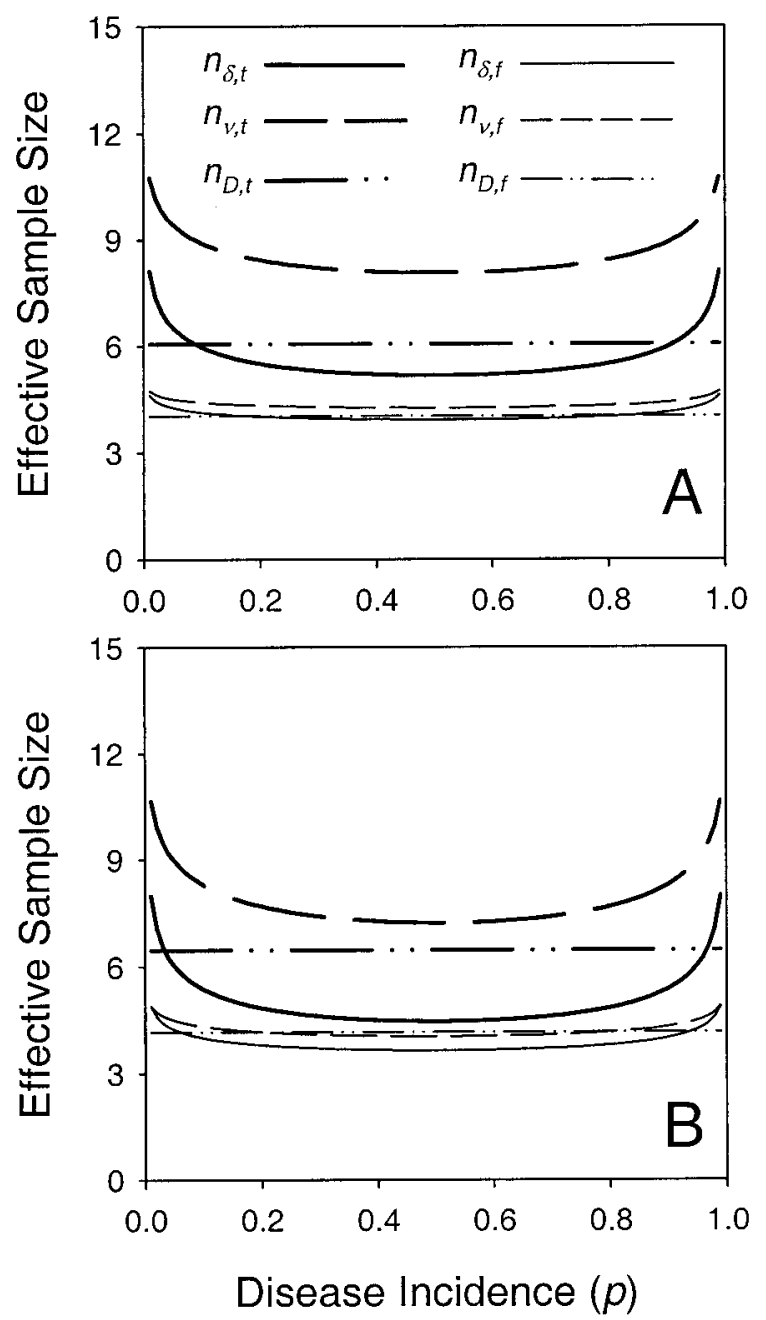

Fig. 2. Relationships between three different effective sample sizes- $n_{D}\left(n_{D, t}\right.$ or $n_{D, f}$; equation 8$), n_{\delta}\left(n_{\delta, t}\right.$ or $n_{\delta, f}$; equation 9$)$, and $n_{v}\left(n_{v, t}\right.$ or $n_{v, f}$; equation 10) - and the estimated parameters of the beta-binomial distribution for probability of disease at the leaflet and leaf scales, $\hat{p}_{t}$ an $\hat{p}_{f}$, respectively, in A, 1996 and B, 1997. Values were calculated using the estimated parameters of the binary power law (equation 6) reported in Turechek and Madden (25), namely $\hat{a}_{t}=0.235, \hat{b}_{t}=1.14$ for 1996 leaflets; $\hat{a}_{t}=0.287$, $\hat{b}_{t}=1.18$ for 1997 leaflets; $\hat{a}_{f}=0.273, \hat{b}_{f}=1.05$ for 1996 leaves; and $\hat{a}_{f}=0.31$, and $\hat{b}_{f}=1.09$ for 1997 leaves. The values of $n_{D}$ were calculated with mean index of dispersion $(D)$, namely $D_{t}=2.48$ for 1996 leaflets; $D_{t}=$ 2.32 for 1997 leaflets; $D_{f}=1.24$ for 1996 leaves; and $D_{f}=1.20$ for 1997 leaves. function has broad application because, unlike the beta-binomial function, the modified binomial function is algebraically easy to manipulate in order to explore the interrelationship of $\hat{p}_{f}, \hat{p}_{t}, \hat{\theta}_{f}$, and $\hat{\theta}_{t}$, the latter two through effective sample sizes $n_{\bullet, f}$ or $n_{\bullet, t}$ (equations 8 to 10 and Fig. 2).

The methodology developed here, and applied to the strawberry leaf blight system, has applicability in sampling for disease incidence $(4,5,16,17)$. When spatial information is available across a range of scales, it is possible to make predictions from any scale to any other scale. This can be particularly useful, for example, in orchard systems where incidence of fruit infection is of interest. Clearly, sampling is easiest at the tree level, but the relationship between incidence of diseased trees, the highest scale, and diseased fruit (i.e., the lowest scale) is such that $100 \%$ incidence of trees corresponds to very low levels of incidence at the fruit level. Sampling clusters of fruit on branches would probably provide the level of resolution necessary to make accurate predictions at the fruit level. That is, one would determine the proportion of branches where at least one fruit was infected and predict the proportion of infected fruit. However, because disease incidence at the branch level is probably heterogenous (relative to the binomial), this heterogeneity must be accounted for in order to obtain accurate estimates at the lower scale.
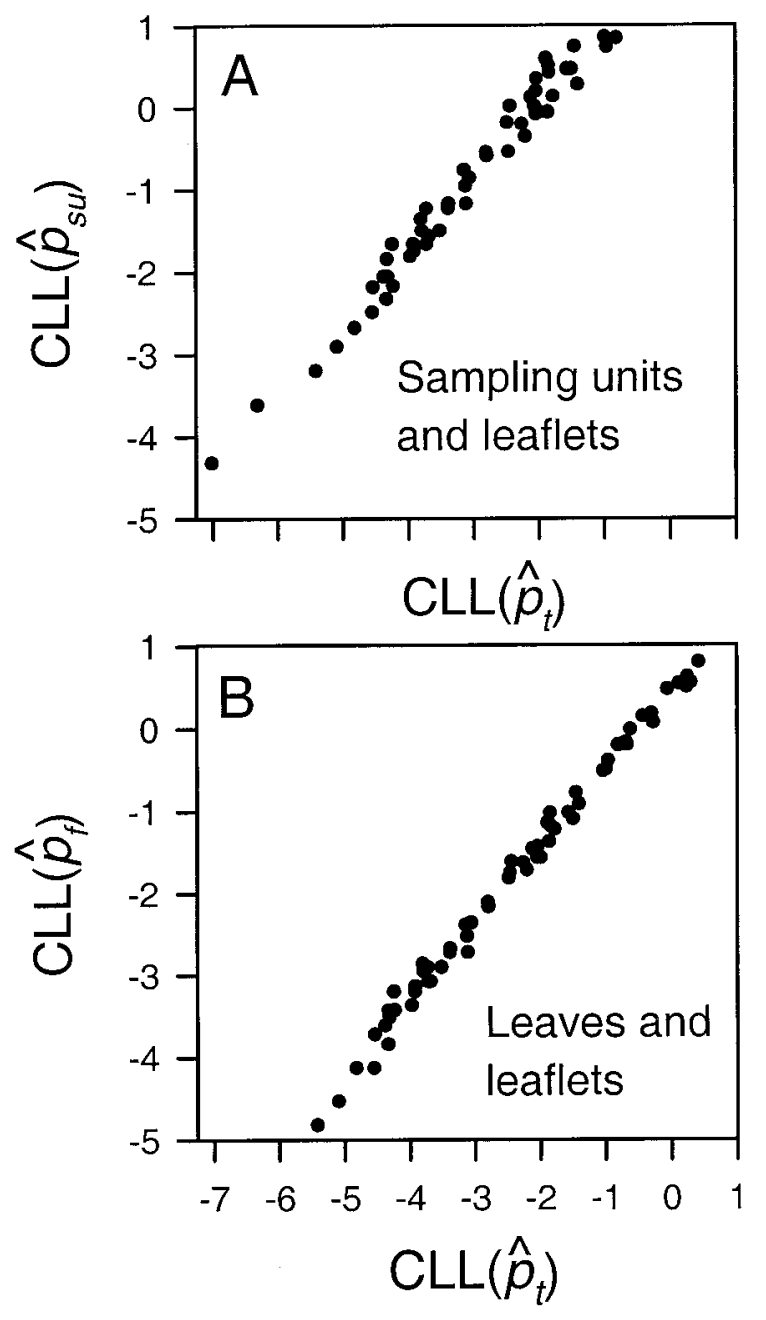

Fig. 3. A, Relationship between the complementary log-log (CLL) transformation of estimated parameter of the beta-binomial distribution at the lowest (leaflet; $t$ ) scale, representing probability of disease $\left[\operatorname{CLL}\left(\hat{p}_{t}\right)\right]$, and the CLL transformation of estimated binomial probability that a sampling unit has one or more diseased leaflets $\left[\operatorname{CLL}\left(\hat{p}_{s u}\right)\right]$ for 1996. B, Relationship between $\operatorname{CLL}\left(\hat{p}_{t}\right)$ and the estimated parameter of the beta-binomial distribution at the intermediate (leaf; $f$ ) scale $\left[\operatorname{CLL}\left(\hat{p}_{f}\right)\right]$ for 1996. 
To begin to develop such a sampling procedure it is first necessary to rearrange equation 18 so incidence at the lowest scale is written in terms of incidence at the intermediate scale. That is,

$$
\tilde{p}_{t}=1-\left(1-\hat{p}_{f}\right)^{n_{\bullet}, f} / n_{0}, t
$$

In order to estimate leaflet disease incidence with a desired level of precision, an estimate of the variance is needed (16). Following Hughes et al. (9), an approximate expression for the variance of $\tilde{p}_{t}$ can be calculated from

$$
\widetilde{v}_{t}=\left(\frac{\mathrm{d} \tilde{p}_{t}}{\mathrm{~d} \hat{p}_{f}}\right)^{2} \cdot v_{f}
$$

in which $v_{f}$ is the variance of disease incidence at the leaf scale (observed or theoretical). The standard error is then $\operatorname{SE}\left(\widetilde{p}_{t}\right)=\sqrt{\widetilde{v}_{t} / N}$. This standard error could be used in any number of models for determining sample size; the specific equation is dependent upon the sampling procedure (e.g., fixed sample size or sequential sampling plans) and how precision is defined (e.g., by the coefficient of variation). As a first-step approximation in determining $\tilde{v}_{t}$, one can assume that diseased leaves have a binomial distribution. This is reasonable based on the (relatively) low level of heterogeneity at the leaf scale $(25,26)$, with corresponding effective sample size being close to 5 over most of the range of disease incidence (2). If effective sample size at the leaflet scale was fixed (i.e., $n_{\bullet, t}$ is not a function of incidence, such as with case iii), then one can show that the variance at leaflet scale is given by

$$
\widetilde{v}_{t}=\frac{\hat{p}_{f} n_{f}\left(1-\hat{p}_{f}\right)^{\left(2 n_{f}-n_{\bullet, t}\right) / n_{\bullet}, t}}{n_{\bullet, t}^{2}}
$$

For more accurate descriptions of the relationship between heterogeneity and disease incidence at the lowest scale, more complicated expressions will have to be considered. Moreover, the error in estimating parameters (including the effective sample sizes) and the error in use of equation 18 for describing the $\hat{p}_{f}: \hat{p}_{t}$ relationship (relative to a perfect relationship) should be considered. This will require extensive analytical and simulation research to predict, ultimately, the standard error of the estimated disease incidence at the intermediate scale. The procedures presented by Jones (10) for binomial sampling provide a good guide for interested researchers.

It should be pointed out that the observed $\hat{p}_{f}: \hat{p}_{t}$ relationship (Fig. 4) is just one of many possible, depending on the arrangement of diseased leaflets among leaves (within sampling units) (26). The actual arrangement for a given data set is reflected by the values of $\hat{p}_{f}$ and $\hat{\theta}_{f}$, conditional on the realized $\hat{p}_{t}$ and $\hat{\theta}_{t}$, which in turn, determines $n_{\bullet, f}$ and other metrics for heterogeneity at the intermediate scale (e.g., $D_{f}$ ). Thus, successful prediction of $\hat{p}_{f}$ from $\hat{p}_{t}$ (equation 18), or $\hat{p}_{t}$ from $\hat{p}_{f}$ for group-sampling purposes $(4,5)$, requires information (direct or indirect) on the arrangement of diseased leaflets among leaves within sampling units, as quantified in this paper. In general, the observed $\hat{p}_{f}$ and $\hat{\theta}_{f}$ for Phomopsis leaf blight $(25,26)$ are on the low end of possible values given the achieved values of $\hat{p}_{t}$ and $\hat{\theta}_{t}$. As discussed in detail by Turechek and Madden (26), this indicates a very high degree of clustering of diseased leaflets within sampling units, a direct reflection of the putative short-range spore-dispersal mechanism for $P$. obscurans. Therefore, the estimates of the effective sample sizes, using the methods in this paper, provide direct information on the clustering of diseased individuals within sampling units, conditional on the incidence and spatial pattern of disease at the lowest scale. Although it may be possible to assume a random distribution for the intermediate scale for some predictive cases (because of the low degree of heterogeneity at the leaf scale [26]), more accurate predictions require specific information on the heterogeneity of disease at both scales. Once this prior information is known, one can accurately relate disease incidence among at least three hierarchical scales, as shown here.

An effective sample size (e.g., $n_{\bullet, t}$ ) can be thought of as a way to account for the loss of information about disease incidence through the effects of aggregation or clustering (15). Because the assumption of independence is not valid when diseased individuals are aggregated, each individual leaflet contributes less than one "unit" of information in determining incidence at the highest scale; this is analogous to there being less than $n_{t}$ indi-
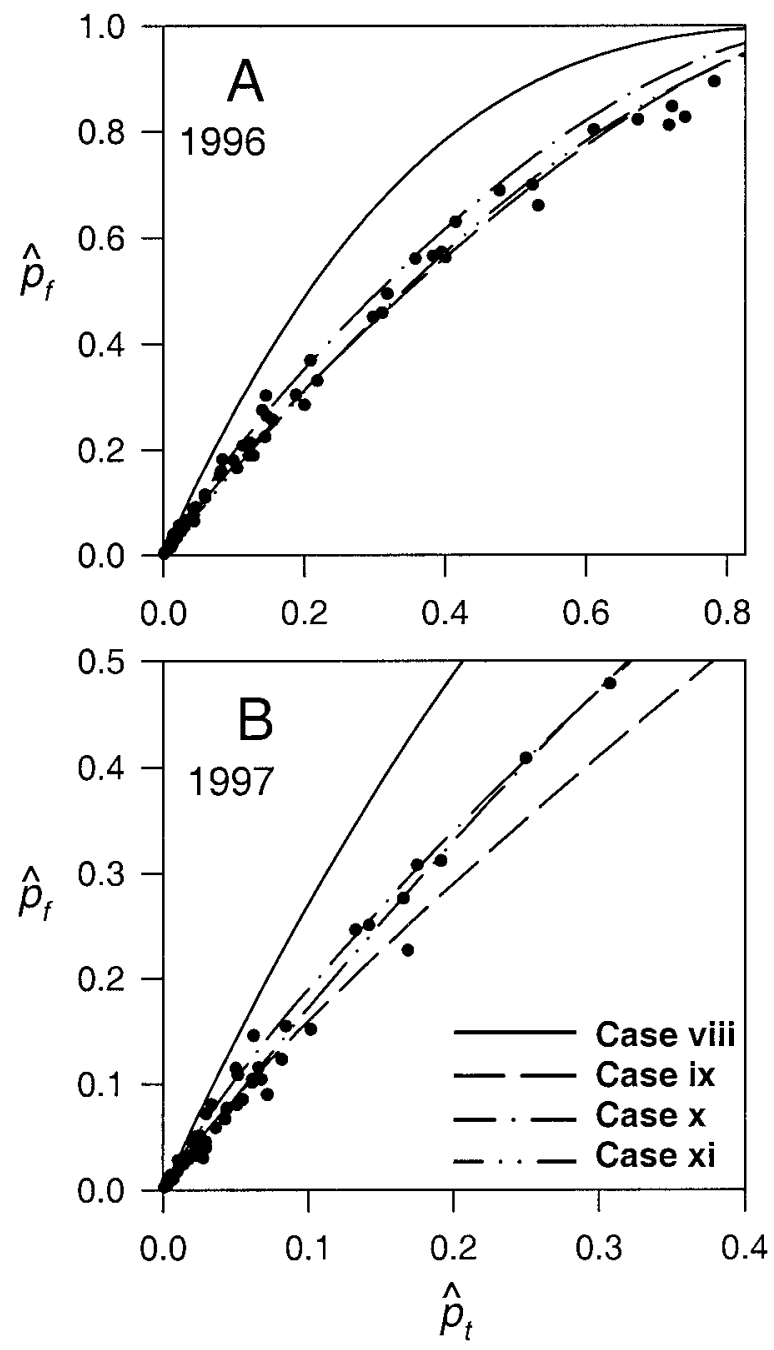

Fig. 4. Relationships between estimated parameters of the beta-binomial distribution for probability of disease at the leaflet and leaf scales, $\hat{p}_{t}$ and $\hat{p}_{f}$, respectively, in A, 1996 and $\mathbf{B}, 1997$. Observed data are shown as points and represent single field assessments of Phomopsis leaf blight in strawberry fields. Case viii (solid line) was derived by assuming that incidence could be described by the binomial distribution at both the leaflet and leaf scales (equation 17). In case ix (broken line), leaf disease incidence was assumed to be random (i.e., binomial [equation 3]) and leaflet disease incidence was assumed to be described by the beta-binomial distribution with heterogeneity quantified by the effective sample size of Madden and Hughes, $n_{v, t}(15)$. Heterogeneity varied with incidence at the leaflet scale according to the binary power law (equation 6) (such that $n_{\bullet, t}$ in equation 18 was given by equation 10 [i.e., $n_{v, t}$, with $\hat{\theta}_{t}$ given by equation 5 , using the parameters $\hat{a}_{t}=$ $0.235, \hat{b}_{t}=1.14$ for 1996 ; and $\hat{a}_{t}=0.287, \hat{b}_{t}=1.18$ for 1997]), and by the binomial distribution at the leaf scale (using $n_{f}=5$ for $n_{\bullet, f}$ in equation 18). The binary power law estimates were obtained by fitting equation 6 to the data. In case $\mathrm{x}$ (dash-dot line), incidence was assumed to be described by the beta-binomial distribution, with heterogeneity varying at the leaflet level according to $n_{v, t}$ (same as case ix) and constant heterogeneity at the leaf scale using the effective sample size defined in equation $8\left(n_{D, f}\right)$. In case xi (dashdot-dot line), curve fitting was used to estimate the intercept parameter $\ln \left(n_{t} / n_{f}\right)$ in equation 19 and assumed a constant slope of 1 . 
viduals in the sampling unit. Thus, fewer sampling units are diseased at a given level of incidence at the lowest scale than expected for a random (i.e., binomial) situation where individuals are independent. Use of equation 11 is an attempt to approximately correct for this aggregation. This concept can be expanded for relating disease incidence at an intermediate and lower scale (equations 16 and 18). For our study, the value $n_{t} / n_{f}=3$ can be thought of as a ratio that represents the maximum amount of information that can be obtained from the data when we wish to estimate $p_{f}$ from $p_{t}$. A value less than 3 , because of aggregation, results in lower disease incidence at the leaf scale than expected for a random pattern of disease (i.e., when disease leaflets are independent). Consider case ix as an example, where $n_{v, t}$ is used for the lowest scale and $n_{f}(=5)$ for the intermediate scale. If we use the calculated value of $n_{v, t}$ when $p_{t}=0.5$ (Fig. 2), the incidence at which degree of heterogeneity is assumed to be at a maximum, then $n_{v, t} / n_{f}=8.06 / 5=1.61$ for 1996 and $7.22 / 5=1.44$ in 1997. With case $\mathrm{x}$, in which $n_{D, f}$ is substituted for $n_{f}$ in equation 18 , values of 1.99 and 1.75 are obtained for the ratio in 1996 and 1997 , respectively. With curve fitting (case xi), $n_{v, t} / n_{f}$ is estimated directly from the data relating the two scales (Fig. 4), and estimates of 1.67 and 1.80 for 1996 and 1997, respectively, are obtained. Thus, when we account for aggregation, the ratio is about 50 to $60 \%$ of the nominal value of 3 for the binomial distribution.

In conclusion, the modeling approach of Hughes et al. (9) and Madden and Hughes (15) could be extended to a three-scaled hierarchical system. Although there are many possible values for incidence at an intermediate scale for a given incidence at the lowest scale (26), the relationship between incidence at the highest scale (sampling unit) and each of the lower scales could be exploited to accurately predict the relationship in incidence between the intermediate and lowest scales. This required the use of the approximation models of Hughes and Gottwald $(4,5)$ and Madden and Hughes (15), with or without curve fitting, to provide the linkage between scales. The most accurate models comprised a binomial-type function with an effective sample size term that depends on disease heterogeneity (assumed or measured) at each scale. In the case of Phomopsis leaf blight of strawberry, the incidence-scale relationship was best described when the effective sample size was based on the parameters of the binary power law at the lowest scale in the hierarchy.

\section{LITERATURE CITED}

1. Aitkin, M., Anderson, D., Francis, B., and Hinde, J. 1989. Statistical Modelling in GLIM. Clarendon Press, Oxford.

2. Chen, C. L., and Swallow, W. H. 1990. Using group testing to estimate a proportion, and to test the binomial model. Biometrics 46:1035-1046.

3. Farrington, C. P. 1992. Estimating prevalence by group testing using generalized linear models. Stat. Med. 11:1591-1597.

4. Hughes, G., and Gottwald, T. R. 1998. Survey methods for assessment of citrus tristeza virus incidence. Phytopathology 88:715-723.

5. Hughes, G., and Gottwald, T. R. 1999. Survey methods for assessment of citrus tristeza virus incidence when Toxoptera citricida is the predominant vector. Phytopathology 89:487-494.

6. Hughes, G., and Madden, L. V. 1992. Aggregation and incidence of disease. Plant Pathol. 41:657-660.

7. Hughes, G., and Madden, L. V. 1995. Some methods allowing for aggregated patterns of disease incidence in the analysis of data from designed experiments. Plant Pathol. 44:927-943.

8. Hughes, G., Madden, L. V., and Munkvold, G. P. 1996. Cluster sampling for disease incidence data. Phytopathology 86:132-137.

9. Hughes, G., McRoberts, N., Madden, L. V., and Gottwald, T. R. 1997. Relationship between disease incidence at two levels in a spatial hierarchy. Phytopathology 87:542-550.

10. Jones, V. P. 1994. Sequential estimation and classification procedures for binomial counts. Pages 175-205 in: Handbook of Sampling Methods for Arthropods in Agriculture. L. P. Pedigo and G. D. Buntin, eds. CRC Press, Boca Raton, FL.

11. Kish, L. 1965. Survey Sampling. Wiley-Interscience, New York.

12. Kotliar, N. B., and Weins, J. A. 1990. Multiple scales of patchiness and patch structure: A hierarchical framework for the study of heterogeneity. Oikos 59:253-260.

13. Maas, J. L. 1998. Compendium of Strawberry Diseases. 2nd ed. The American Phytopathological Society, St. Paul, MN.

14. Madden, L. V., and Hughes, G. 1994. BBD-computer software for fitting the beta-binomial distribution to disease incidence data. Plant Dis. 78:536-540.

15. Madden, L. V., and Hughes, G. 1999. An effective sample size for predicting plant disease incidence in a spatial hierarchy. Phytopathology 89:770-781.

16. Madden, L. V., and Hughes, G. 1999. Sampling for plant disease incidence. Phytopathology 89:1088-1103.

17. Madden, L. V., Hughes, G., and Ellis, M. A. 1995. Spatial heterogeneity of the incidence of grape downy mildew. Phytopathology 85:269-275.

18. Maynard Smith, J. 1968. Mathematical Ideas in Biology. Cambridge University Press, London.

19. Nachman, G. 1981. A mathematical model of the functional relationship between density and spatial distribution of a population. J. Animal Ecol. 50:453-460.

20. Nyrop, J. P., and Binns, M. R. 1990. Quantitative methods for designing and analyzing sampling programs for use in pest management. Pages 67132 in: Handbook of Pest Management in Agriculture. D. Pimentel, ed. CRC Press, Boca Raton, FL.

21. Perry, J. N. 1987. Host-parasitoid models of intermediate complexity. Am. Nat. 130:955-957.

22. Rao, J. N. K., and Scott, A. J. 1992. A simple method for the analysis of clustered binary data. Biometrics 48:577-585.

23. Seem, R. C. 1984. Disease incidence and severity relationships. Annu. Rev. Phytopathol. 22:133-150.

24. Swallow, W. H. 1985. Group testing for estimating infection rates and probabilities of disease transmission. Phytopathology 75:882-889.

25. Turechek, W. W., and Madden, L. V. 1999. Spatial pattern analysis of strawberry leaf blight in perennial production systems. Phytopathology 89:421-433.

26. Turechek, W. W., and Madden, L. V. 2001. Effect of scale on plant disease incidence and heterogeneity in a spatial hierarchy. Ecol. Model. 144:77-95.

27. Wilson, L. T., and Room, P. M. 1983. Clumping patterns of fruit and arthropods in cotton, with implications for binomial sampling. Environ. Entomol. 12:50-54.

28. Xu, X.-M., and Madden, L. V. 2002. Incidence and density relationships of powdery mildew on apple. Phytopathology 92:1005-1014. 